\title{
Gradient heat flux measurement during condensation at the surfaces of pipes
}

\author{
Sergey Sapozhnikov ${ }^{1}$, Vladimir Mityakov ${ }^{1}$, Alexander Babich ${ }^{1 *}$, and Elza Zainullina ${ }^{1}$ \\ ${ }^{1}$ Peter the Great St. Petersburg Polytechnic University, 195251, 29 Politekhnicheskaya st., Saint-Petersburg, Russia
}

\begin{abstract}
Gradient heat flux measurement was used to study steam condensation at the inner and outer surfaces of pipes. Experimental setups were developed, manufactured and tested. The setups were able to incline the pipes for different angles relative to vertical and to rotate them around their main axes. Local heat transfer coefficients (HTC) along the pipe length and perimeter were determined. Formation and motion of condensate film were studied. The results are corresponding to classical ideas and give us some new information.
\end{abstract}

\section{Introduction}

Almost all the works on heat transfer during condensation are based on classical model proposed by V. Nusselt in 1916 [1]. It is based on the assumptions corresponding to laminar condensate film flow along a vertical surface.

Nusselt equation is valid for stationary steam flow, that is why HTC equations for non-stationary condensation based on Nusselt formula include correlation factors. In 1948 Kapitsa put forward the correlation factor for condensate film waviness [2]. Wunsch showed the correlation factor for non-isothermal condensate film [3]. Labuntsov studied heat transfer during condensation at non-isothermal surfaces and proposed correlation in form of dependence of Nusselt number from Reynolds number [4]. Also, Labuntsov and Voskresensky introduced a correction for variability of liquid properties [5].

The first analytical solution for condensation at inclined surface was presented by Hasan and Jacob [6], where the laminar flow of condensate film along the outer surface of the inclined pipe under the action of gravity was considered. Kamminga modified this formula and published his own solution [7]. These formulae are huge and do not allow to estimate local HTCs.

The basic method of experiments is thermometry. The heat flux is measured as average one, without estimating of its value per unit area due to the lack of heat flux sensors. The first experimental works dealt with condensation inside vertical pipes were published by Icob in 1935 [8]. After the Fukushima Daiichi nuclear disaster, many new experimental works on film condensation inside and outside the pipes were published. Scientific groups study the effect of noncondensing gases $[9,10]$, apply coatings that allow to maintain droplet condensation [11], develop surfaces for heat transfer enhancement $[12,13]$.

There are few experimental works on study of condensation at the surface of a single horizontal pipe. More often, horizontally arranged bundles of pipes are considered [14, 15]. Heat transfer at the surface of a single horizontal pipe is usually studied by numerical simulation methods [16, 17]. The basic method of thermometry has several irreparable drawbacks.

Now we propose gradient heat flux measurement for study of heat transfer during condensation for the first time. This method is based on using of gradient heat flux sensors (GHFS) [18]. GHFSs were already used in study of diesel engines $[19,20]$ and convective heat transfer $[21,22]$.

\section{Method}

The results from two different setups are shown. One of them allows to investigate condensation at the inner surface of the pipe, and another one at the outer surface of the pipe.

\subsection{Gradient heat flux sensors}

GHFS generates the electromotive force, which is linearly related to the average heat flux through the cross-section per unit area:

$$
E=S_{0} \cdot F \cdot q
$$

\footnotetext{
* Corresponding author: babich1994@mail.ru
} 
Here, $E$ is the electromotive force, $S_{0}$ is the GHFS sensitivity, $F$ is the GHFS area, $q$ is heat flux per unit area (fig. 1).

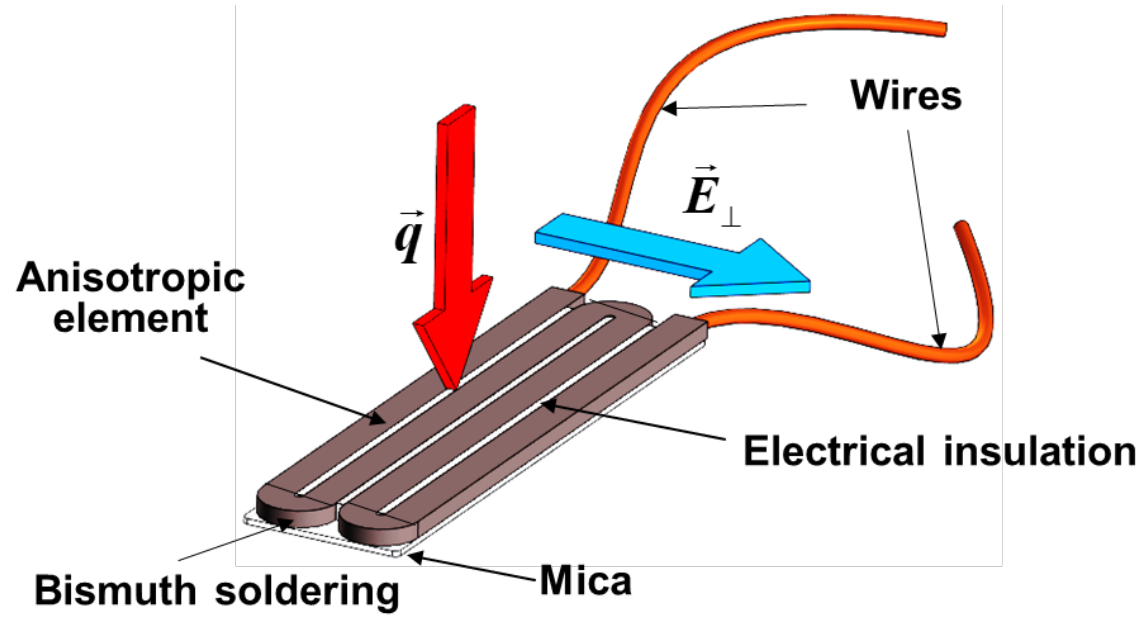

Fig. 1. GHFS schema.

\subsection{Condensation at the outer surface of the pipe}

Stainless steel pipe with length of $1000 \mathrm{~mm}$ and diameter of $20 \mathrm{~mm}$, with the wall thickness of $2 \mathrm{~mm}$ was used as the main element of the setup.

GHFS positioning should minimally distort the condensate film flow. Therefore five cuts for the sensors of $18 \times 3 \times 0,3 \mathrm{~mm}$ were made. The first one was located at
$100 \mathrm{~mm}$ from the upper edge of the pipe, the second at $300 \mathrm{~mm}$, the third at $500 \mathrm{~mm}$, the fourth at $700 \mathrm{~mm}$, and the fifth at $800 \mathrm{~mm}$.

GHFSs were installed on the insulating mica film with a thickness of $10 \mu \mathrm{m}$ and fixed in the cuts with epoxy resin to avoid of electric contact between the sensor and the steel pipe. The resin was applied along the perimeter of the GHFSs, which also excluded electric contact.

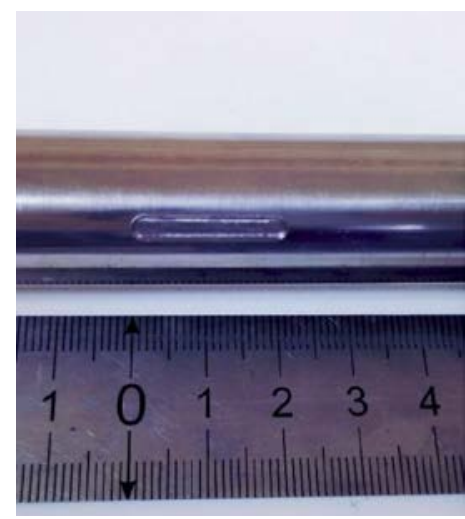

a)

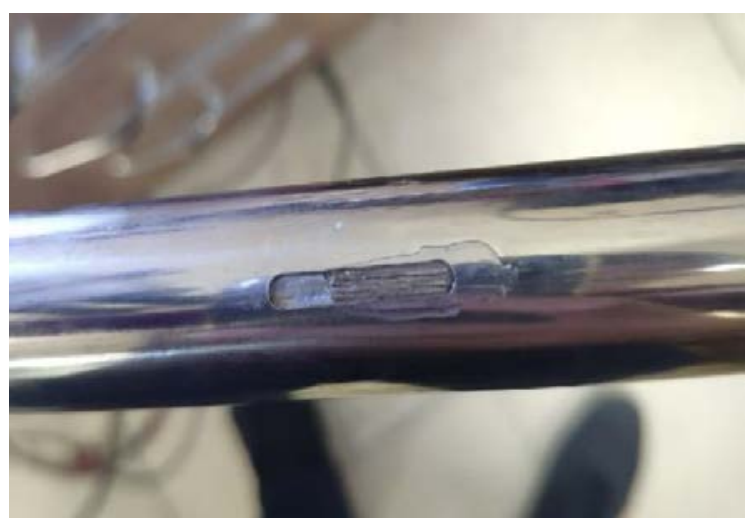

b)

Fig. 2. The cut (a) and the GHFS in the cut (b).

In the pilot version, J-type thermocouples were used. Their disadvantages were unsatisfactory moisture resistance of their insulation and the lack of fixation of thermoelectrodes in the gap between the pipe and the casing.

The upgraded installation uses semi-artificial thermocouples copper-stainless steel.

The pipe is fixed in the shell with the two rubber plugs with a diameter of $55.5 \mathrm{~mm}$ and a thickness of 30 $\mathrm{mm}$. The nozzles for steam supply and condensate drainage are inserted in the holes of the plugs.
The experimental setup provided the inclination of the pipe in the range from 0 to $90^{\circ}$ with a step of $10^{\circ}$. Also, the pipe was able to rotate around its main axis. That allows to measure e local HTCs for all azimuthal angles.

\subsection{Condensation at the inner surface of the pipe}

The experimental setup was similar to the previous one. The main difference is that GHFSs were installed at the 
inner surface. Four segments were cut out from the pipe with an electrospark machine. The first one was located at the distance of $300 \mathrm{~mm}$ from the upper edge of the pipe, and the next ones at a distance of $200 \mathrm{~mm}$ from each other. The small thickness of the cut allowed to solder the segments back with no gaps at the inner surface of the pipe (figures 3, a, b, c).
The pipe with installed back segments was placed in a plastic cover and fastened by two rubber plugs like at the previous experimental setup. All wires were removed through the upper rubber plug ensuring tightness of the installation. The overall view of the experimental setup is shown in figure $3, \mathrm{~d}$.
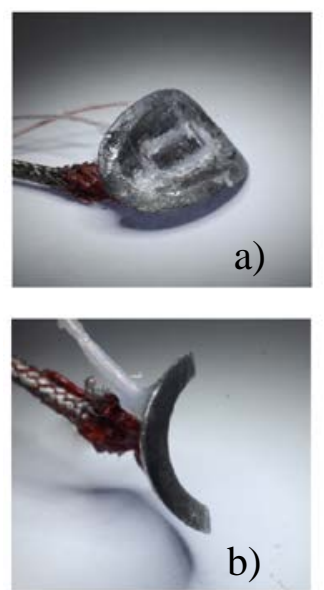

b)

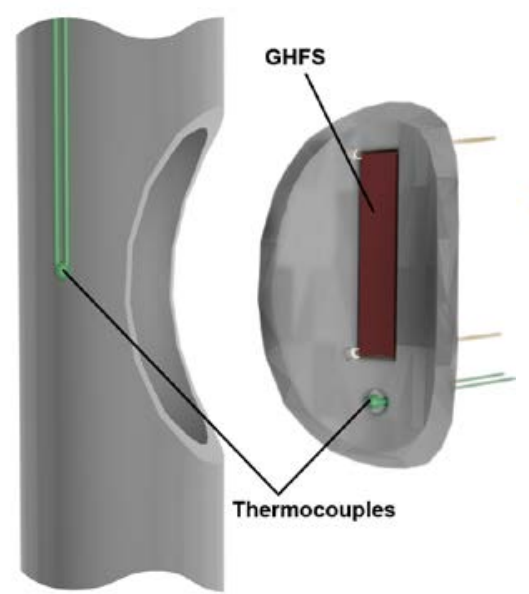

c)

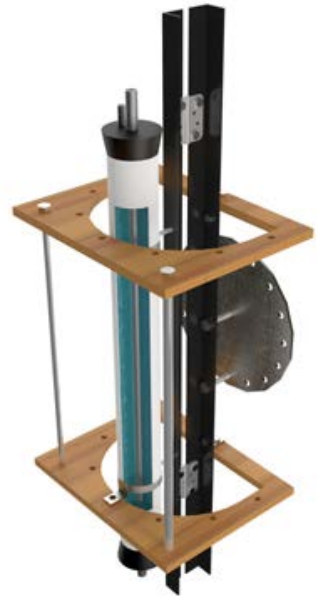

d)

Fig. 3. Segment photo (a, b), GHFS and thermocouple positioning (c), and overall view of the experimental setup (d).

\section{Results and Discussion}

\subsection{Condensation at the outer surface of the pipe}

Figure 4 shows the heat flux diagram from GHFS-2, GHFS-4 and GHFS-5, placed at distances of 300, 700

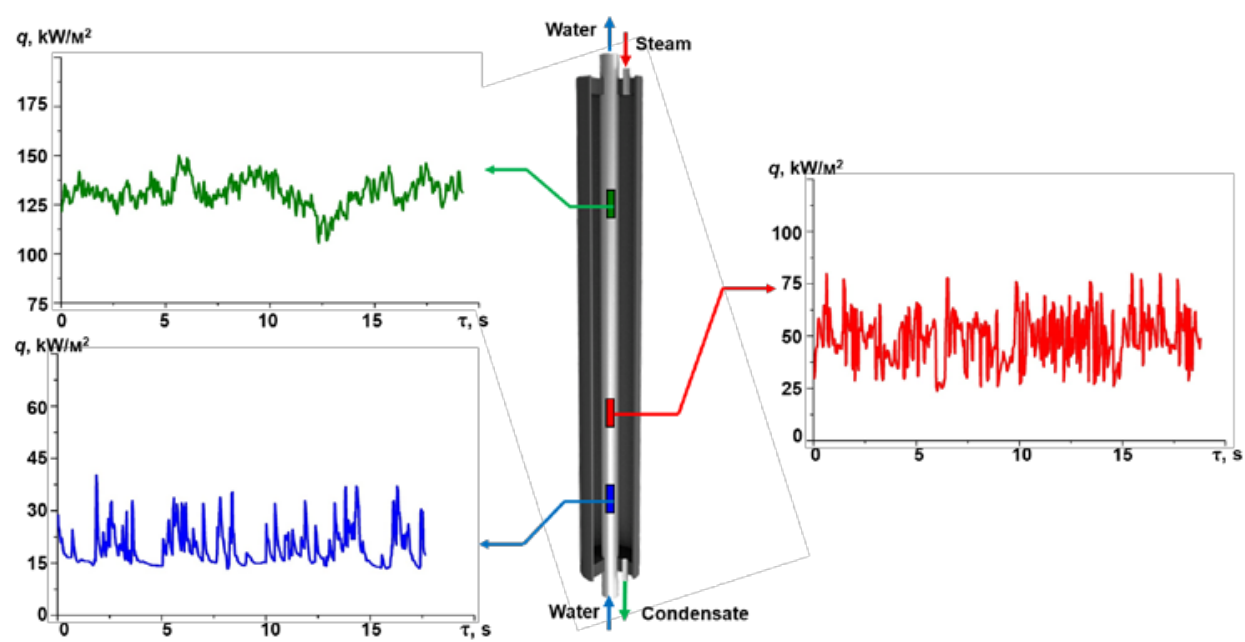

Fig. 4. The heat flux diagram for condensation at the outer surface of the vertical pipe

The pulsations increase at the bottom of the pipe, which is noticeable at the signals of GHFS-2 and GHFS4. Almost all mass of steam was condensed in the upper part of the pipe, so GHFS-5 picked up the absence of a condensate film, which corresponds to the sections of the heat flux diagram with a constant heat flux per unit area. and $800 \mathrm{~mm}$ from the upper edge during condensation at the vertical pipe. 
from the pulsation of the heat flux per unit area at all angles $\varphi$. The highest average heat flux is at the angle of $\varphi=60^{\circ}$. Pulsations increase at the lower sector of the pipe, which is obvious at the heat diagram at $\varphi=150^{\circ}$.

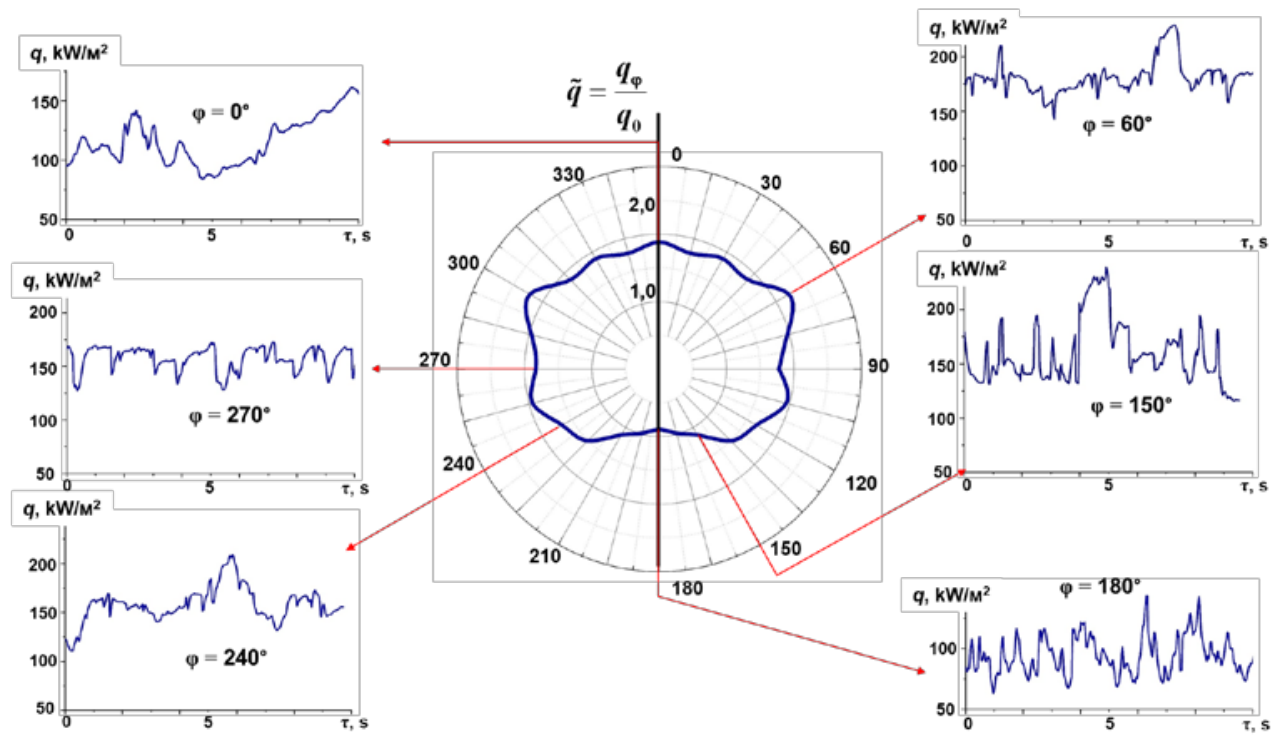

Fig. 5. The dependence of relative heat flux from the angle $\varphi$ in the horizontal pipe.

\subsection{Condensation at the inner surface of the pipe}

The heat flux diagram for condensation at the inner surface of the vertical pipe is shown in figure 6 , a. There are some pulsations of heat flux at GHFS-3 and GHFS-4 due to the wave formation of the condensate film.

Figure 6, b shows heat flux diagrams for GHFSs and HTCs calculated from the thermocouple readings. It is obvious that thermocouples do not completely fix fluctuations of heat flux, therefore, they do not give a complete picture of heat transfer during condensation.

Condensation inside horizontal tubes was also studied. The dependence of HTC coefficient on azimuthal angle at different sections of the pipe is presented in figure 7 . The signals from the GHFS-3 and GHFS-4 show that condensate film flows at downside of the pipe. That is why increasing of the condensate film thickness decreases the HTC.

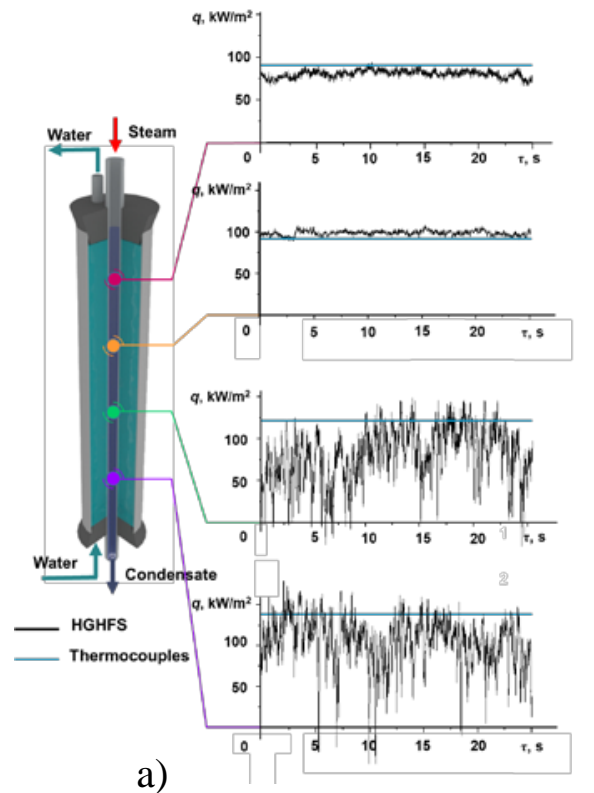

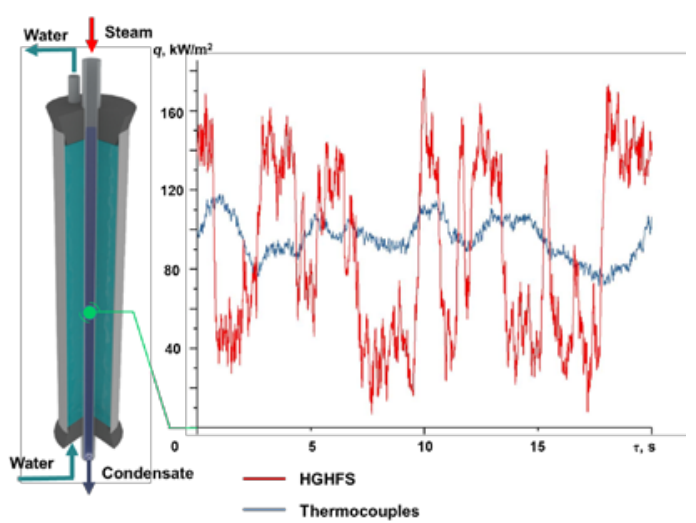

b)

Fig. 6. The heat flux diagram for condensation at the inner surface of the vertical pipe (a) and detailed heat flux diagram for GHFS-3 (b). 


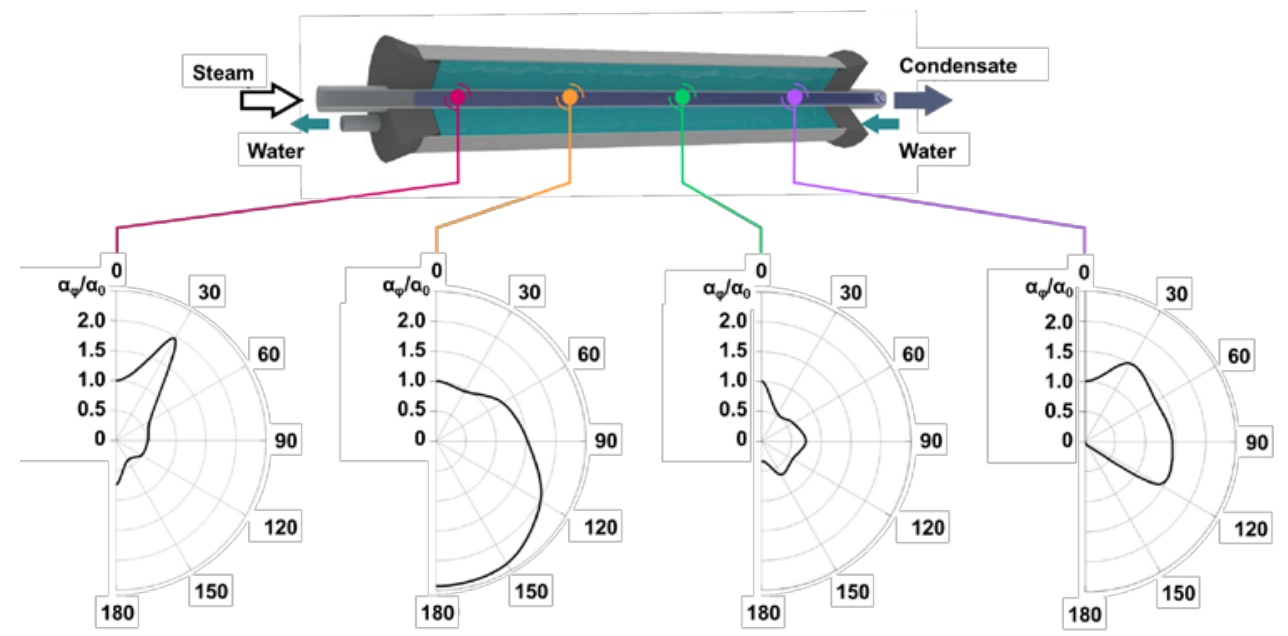

Fig. 7. The heat flux diagram during condensation at the inner surface of the horisontal tube.

\section{Conclusions}

The experimental setups for study of condensation with gradient heat flux sensors have been developed, manufactured and tested.

The advantage of heat flux measurement over thermometry is revealed. New possibilities in the study of condensation with GHFS are shown.

Gradient heat flux measurement will be productive in a wider range of problems related to condensation of steam inside and outside pipes with the with a standard uncertainty of not more than 10\% (ISO/IEC Guide 981:2009)

The reported study was funded by RFBR, project number 17-08-01143 A.

\section{References}

1.W. Nusselt, Z. Ver. Dtsch. Ing. 60(27), 541-546

2.P.L. Kapitsa, Zh. EksperimTeor. Fiz. 18 (3), (1948)

3. G. Wunsch. Freiberger Forschungshefte Bd A, 517, 19- 60 (1973)

4.D.A. Labuntsov, Heat power engineering. 2 (1957)

5.K. D. Voskresensky, Izvesia USSR, OTN. 7 (1948)

6. K. Hassan, M. Jacob, Trans. ASME 80, 4 (1958)

7.W. Kamminga, Heat and Mass Transfer, 23, 9 (1980)

8. M. Icob, Phis. Zeitschrift, 3 (1935)

9.G. Fan, P. Tong, Z. Sun, Y. Chen, Annals of Nuclear Energy 113, 139-146 (2018)

10. M. L. Corradini Nuclear Technology 64(2), 186-195 (1984)

11. D. J. Preston, Z. Lu, Y. Song, Y. Zhao, K. L.Wilke, D. S. Antao, N. Wang Scientific Reports 8 (2018)

12. T. Gebauer, A. R. Al-Badri, A. Gotter-barm, J. El Hajal, A. Leipertz, A. P. Fröba, Int. J. Heat Mass Transf 56(1-2), 516-524 (2013)

13. A. Briggs, S. Sabaratnam, Int. J. Energy Res 27 301-314 (2003)
14. H. W.Hu, G. H. Tang, D. Niu Applied Thermal Engineering. 100 699-707 (2016)

15. J. X. Zhang, L. Wangb International Journal of Heat and Mass Transfer 112 676-688 (2017)

16. G. H. Tang, H. W. Hu, Z. N. Zhuang, W. Q. Tao, Appl. Therm. Eng. 36 414-425 (2012)

17. C.-K. Chen, Y.-T. Lin, Appl. Math. Model, 33(4) 1944-1956 (2009)

18. A V. Mityakov, S. Z. Sapozhnikov, V. Y. Mityakov, A. A. Snarskii, M. I.Zhenirovsky, J. J. Pyrhönen, Sensors Actuators A Phys. 176, 1-9 (2012)

19. S. Z. Sapozhnikov, V.Yu. Mityakov, A. V. Mityakov, A. V. Vintsarevich, D. V.Gerasimov, A. V. Pavlov, L. A. Vorobyev, A. V. Larin, Thermal Processes in Engineering 166-170 (2018)

20. S Z Sapozhnikov et al, J. Phys.: Conf. Ser. 891, 012096 (2017)

21. A. Mityakov, A. Babich, A. Bashkatov, A. Gusakov, A. Dymkin, E. Zainullina, S.Sapozhnikov, V. Mityakov, V. Seroshtanov, MATEC Web of Conferences. "33rd Siberian Thermophysical Seminar, STS 2017" 02006 (2017)

22. A. Mityakov, V. Mityakov, S. Sapozhnikov, A. Kossolapov, D. Markovich, International Conference on Mechanics - Seventh Polyakhov's Reading C. 7106759 (2015) 